\title{
Clinical evaluation of a screen pneumotachograph as an in-line filter
}

\author{
H. Normand*,\#, F. Normand" ${ }^{\#}$ X. Le Coutour", M-A. Metges ${ }^{+}$and A. Mouadil ${ }^{\#}$
}

\begin{abstract}
The American Thoracic Society/European Respiratory Society Task Force underlined that the use of in-line filters during respiratory function tests "is an area of controversy". The aim of the present study was to measure the contamination occurring during forced expiration downstream from a screen pneumotachograph (SP) with and without an in-line filter (Pall PF30S). A total of $\mathbf{4 0}$ healthy subjects performed eight consecutive maximal expiratory manoeuvres into four sterile apparatuses (A1: no filter, no SP; A2: filter-only; A3: SP-only; A4: filter and SP) in random order. A blood agar plate was fixed downstream from the apparatus. Colonyforming units (CFUs) were counted after $24 \mathrm{~h}$ incubation at $37^{\circ} \mathrm{C}$. Of the 40 plates obtained with each apparatus, 13 were sterile with A1 (range 0-679 CFUs), 25 with A2 (0-49 CFUs), 30 with A3 (0-35 CFUs) and 39 with A4 (one CFU in the only positive plate). A1 versus A2 and also A3 versus A4 gave different values for the CFU number, but $\mathrm{A} 2$ and $\mathrm{A} 3$ showed similar contamination levels.

The authors conclude that: 1) the in-line filter does not perform better than a screen pneumotachograph; 2) it does not eliminate the need to decontaminate the pneumotachograph; and 3) equipment placed downstream from an in-line filter and a screen pneumotachograph is almost protected from contamination.
\end{abstract}

KEYWORDS: Cross infection, prevention and control, respiratory function tests, respiratory tract infections

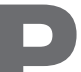

ublic and private healthcare providers are increasingly concerned over control of hospital infections and the limitation of cross-contamination. This preoccupation is particularly acute in cystic fibrosis patients who are colonised with respiratory pathogens $[1,2]$ and in whom pulmonary function tests are routinely performed [3]. Although various circumstantial evidence points to contaminated spirometers being possibly involved in cross-contamination $[4,5]$, there is no direct or even indirect evidence that infection transmission occurs with the use of flow-type systems, which seem less susceptible to bacterial contamination than water-sealed spirometers [6].

Recommendations regarding hygiene in respiratory function testing were restated recently by the American Thoracic Society (ATS)/European Respiratory Society (ERS) Task Force [7]. Prevention of transmission by direct contact can be achieved by disinfecting after each use (or discarding if disposable) mouthpieces, nose clips and any other equipment coming into direct contact with mucosal surfaces. This recommendation appears easy to follow. Transmission of infection can also occur by indirect contact through aerosol droplets, therefore, the ATS/ERS committee recommends that any equipment surface showing visible condensation from expired air should be disinfected or sterilised before re-use. Proximal tubing is easily interchangeable on volume-type spirometers, where contamination occurs most frequently [8, 9], and on certain flow-type pneumotachometers. However, as pointed out by the ATS/ERS Task Force, spirometric equipment also sometimes employs complex valve manifolds whose internal surfaces are exposed to aerosol deposition from expired air. Such equipment is not easy to decontaminate and in some cases it is unrealistic to recommend disinfection after each use. At present, the optimal frequency for disinfection or sterilisation of tubing, valves and manifolds has still not been established.

The appropriate placement of filters with ideally $100 \%$ effectiveness for trapping pathogenic organisms from exhaled air during pulmonary function testing would thus appear to be the most practical method for eliminating transmission of pathogenic organisms between patients [10], and, inasmuch as in-line filters have been shown to be effective in removing pathogens from the expiratory flow [11] without affecting the clinical utility of respiratory function tests [3, 12-14], their use
AFFILIATIONS

*Faculty of Medicine, Physiology Dept, UPRES-EA 3917, University of Caen,

${ }^{\text {\#} F u n c t i o n a l}$ Testing Dept, and

"Hygiene Dept, University Hospital of Caen, Caen, and

${ }^{+}$Functional Testing Dept, University Hospital of Brest, Brest, France.

CORRESPONDENCE

H. Normand

Faculty of Medicine

Physiology Dept UPRES-EA 3917

Avenue de la Côte de Nacre University of Caen

14032 Caen

France

Fax: 33231064871

E-mail: normand-h@chu-caen.fr

Received:

December 162005

Accepted after revision:

June 222006

STATEMENT OF INTEREST

None declared. 
might be encouraged in the clinical laboratory. Patient advocate groups, with the help of their medical committees, have published recommendations including the mandatory use of in-line filters during spirometry, and many laboratory managers have generalised the use of such filters. For instance, a survey of the 19 respiratory function laboratories of the public hospitals of Paris (France) showed that 16 out of the 17 laboratories answering the questionnaire were already using in-line filters for spirometry in 1996, just four years after they came onto the market [15]. However, as explicitly mentioned by the ATS/ERS Task Force [7] and confirmed by a recent exchange of letters [16-18], "the use of such filters is an area of controversy". The ability of these filters to remove bacteria from the high flow produced by true [19] or simulated [20] forced expiration has been seriously questioned and the use of in-line filters does not eliminate the need for regular cleaning and decontamination of lung function equipment. Furthermore, combined with a classical screen pneumotachograph, the least efficient (in terms of bacterial retention) and least resistive of four commercially available specially designed filters [20] already reaches the upper limit of resistance to flow set by the ATS/ERS Task Force guidelines for spirometry measurement $[12,21]$. Finally, while the reduction in filterrelated respiratory parameters is clinically unimportant, it nevertheless depends on the resistance to air flow [22] and is presumably correlated to filtration efficiency. Filters have been shown to reduce forced expiratory volume in one second [12, 14], forced vital capacity [14] and peak expiratory flow [12, 14], indicating that in research and in multicentre studies especially, results obtained with and without a filter should be analysed separately [14].

The most recent lung function equipment is built around a flow-measuring device (usually connected to a pressure transducer) held close to the mouth and so theoretically, although not necessarily [6], it is more exposed to contamination than the inner surface of the bellow of a volume-type spirometer. Manufacturers have developed sensors that are now easier to decontaminate, if not disposable. As standard spirometry can be carried out with just this one sensor (connected to the electronic equipment), it could conceivably be replaced for each patient, or at least for those most at risk from cross-infection, provided that the calibration procedures are not too time-consuming. As pointed out by the ATS/ERS Task Force, difficulties arise when the flow-type spirometer is connected to a more complex system such as those used for plethysmography or gas transfer measurements, where decontamination is a problem. Screen pneumotachographs (Lilly type) are widely used as flow-measuring devices. Made of a fine wire mesh screen with low resistance to air flow, the screen pneumotachograph might also be useful in protecting complex equipment from contamination by pathogens.

The aim of the present study was to measure the natural contamination occurring during forced expiration downstream from a screen pneumotachograph with and without an in-line filter.

\section{MATERIAL AND METHODS Material}

A special coupling device (fig. 1) was made comprising of the following three parts. 1) A screw lid in which a hole has been drilled and a connector has been glued. Two identical lids with different connectors were made because the diameters of the pneumotachograph and the filter were slightly different. 2) A corresponding PVC tubing in which 16 holes $(0.8 \mathrm{~cm}$ in diameter) had been drilled to allow forced expiration. 3) A plastic ring used to maintain the agar plate. The plane of the agar plate was at a distance of $1.5 \mathrm{~cm}$ from the centres of the holes. This device, designed to hold an agar plate, was built in order to test natural contamination occurring during forced expiration in four different situations: in open air with (A2) and without (A1) a filter and downstream from a screen pneumotachograph (fig. 2) with (A3) and without (A4) a filter.

Four apparatuses (A1 to A4) were used to represent the four situations tested (fig. 3), these consisted of the following. A1: Mouthpiece (MP), elbow connector (EC); $\mathrm{L}=16.1 \mathrm{~cm}$; A2: MP, in-line filter (Pall PF30SJE; Pall Corporation, East Hills, NY, USA), EC; $\mathrm{L}=23 \mathrm{~cm}$; A3: MP, EC, screen pneumotachograph (Jaëger PT36; Jaëger, Wursburg, Germany); L=23.4 cm; and A4: MP, in-line filter, EC, screen pneumotachograph; $\mathrm{L}=30.3 \mathrm{~cm}$; with $\mathrm{L}$ equalling distance between the mouthpiece opening and the surface of the agar plate.

Note that the distances between the MP opening and the surface of the agar plate are similar in A2 and A3. The EC is a standard connector used with Jaëger Systems (Ref: 852352; Jaëger). The MP is a disposable plastic one. The Pall PF30SJE filter is the same as the widely used Pall PF30S with connector dimensions adapted to the Jaëger pneumotachograph. This filter was chosen because, whilst one of the commercially available filters offering least resistance, when combined with the screen pneumotachograph used in the current study, it already reaches the limit of resistance recommended by the ATS/ERS Committee [12]. The screen pneumotachograph was used nonheated. A trypticase soy agar plate with 5\% horse blood was fixed downstream from the apparatus in the coupling device (fig. 1)

The expired gas was flushed along the agar plate, and driven through the 16 holes whose total surface $\left(8 \mathrm{~cm}^{2}\right)$ was higher than the minimum surface of the connection tubing $\left(5 \mathrm{~cm}^{2}\right)$ ensuring that the expiration was not limited by the device.

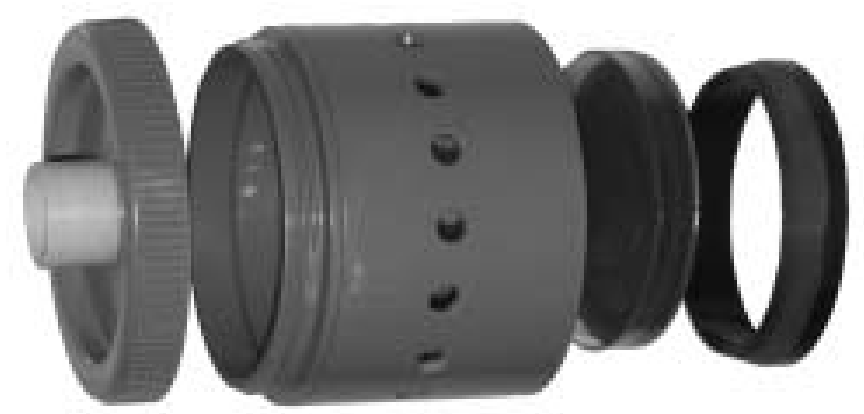

FIGURE 1. The coupling device is made of three parts built from polyvinylchloride (PVC) draining tubing. 1) A screw lid in which a hole has been drilled and a connector has been glued. Two identical lids with different connectors were made because the diameters of the pneumotachograph and the filter were slightly different. 2) A corresponding PVC tubing in which 16 holes $(0.8 \mathrm{~cm}$ in diameter) have been drilled to allow forced expiration. 3) A plastic ring used to maintain the agar plate. The plane of the agar plate was at a distance of $1.5 \mathrm{~cm}$ from the centres of the holes. 


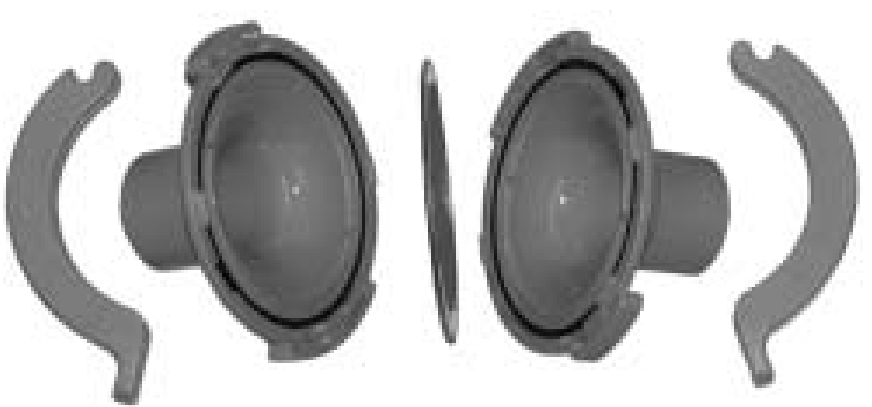

FIGURE 2. Open view of the screen pneumotachograph. Reassembling the system (including positioning of the circular seals) takes $<30 \mathrm{~s}$.

\section{Subjects and protocol}

A total of 40 healthy subjects, students of the Faculty of Medicine, University of Caen (Caen, France), or members of staff, with no history of respiratory disease, gave their informed consent and performed eight consecutive maximal expiratory manoeuvres from total pulmonary capacity into each apparatus, with a 30-s pause between expirations. The study was approved by the regional ethical committee (Consultation Committee for the Protection of People in Biomedical Research, Basse-Normandy). All subjects were trained for pulmonary function testing. They were respiratory technicians, hygienists or medical doctors of the hospital or medical students in pneumology working in the laboratory. The number of expirations was chosen in line with the ATS/ ERS Task Force recommendation, as eight manoeuvres is generally a practical upper limit for most subjects [21]. Immediately before the series of expirations, all the components of the apparatus were reassembled together with a fresh agar plate. All parts (except the pneumotachograph screen) were wiped with alcohol and allowed to dry immediately before reassembly. Handling was done on a table disinfected with Surfanios ${ }^{\circledR}$ (Laboratoire Anios, Hellemmes, France). A dry sterile metallic screen $\left(120^{\circ} \mathrm{C}\right.$ sterilisation) was inserted in the pneumotachograph (A3 and A4) and a new filter was used (A2 and A4). The order of use of the apparatus was randomised according to a Latin square design with 10 identical repetitions. Colony-forming units (CFUs) were counted after $24 \mathrm{~h}$ of incubation at $37^{\circ} \mathrm{C}$ under inspection with a magnifying glass.

\section{Statistical analysis}

The plates were defined as negative (no contamination) or positive ( $\geqslant 1 \mathrm{CFU}$ ) and a Cochran $\mathrm{Q}$ test was performed between the four situations. Individual comparisons between apparatuses were carried out with a McNemar's test. The number of CFUs in the four apparatuses were also compared with a nonparametric test (Friedman test), because the data was not normally distributed; individual comparisons were performed with a Wilcoxon paired-sample test. All the CFUs were counted. However the highest count $(679 \mathrm{CFU}$ in one A1 plate) is approximate as some of the CFU were confluent.

The difference between A1 and A2 represents the filter's ability to prevent contamination of the pneumotachograph. The difference between A3 and A4 represents the filter's ability to prevent contamination of the equipment placed downstream to

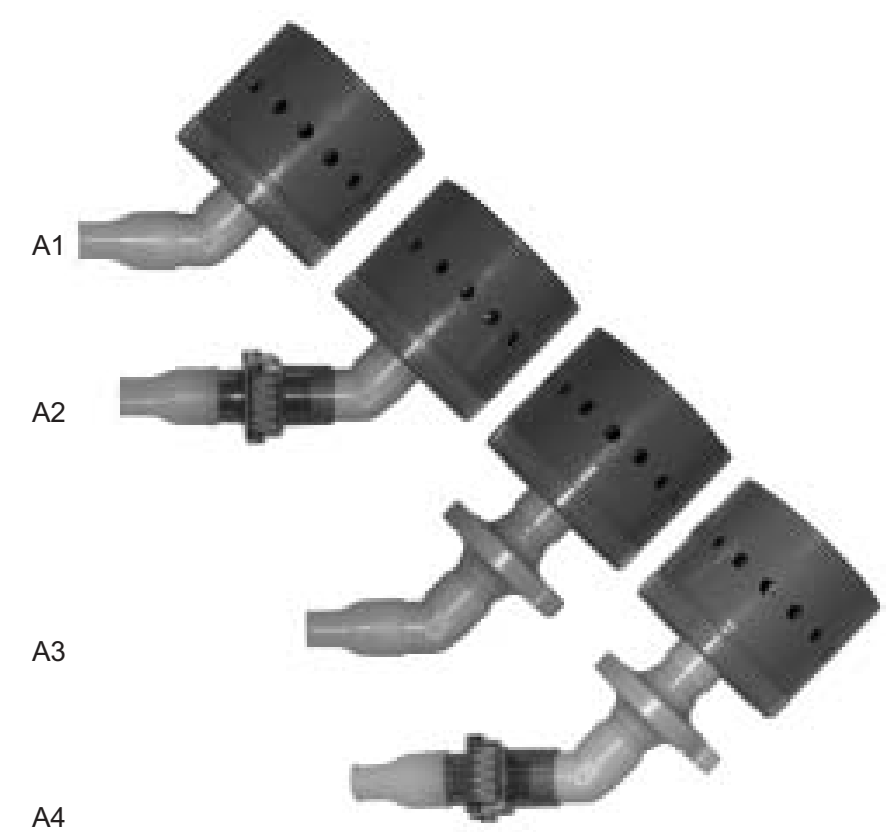

FIGURE 3. From top to bottom: apparatus 1 (A1), from left to right: mouthpiece, elbow connector, coupling device; apparatus 2 (A2), from left to right: mouthpiece, in-line filter, elbow connector, coupling device; apparatus 3 (A3), from left to right: mouthpiece, elbow connector, pneumotachograph, coupling device; and apparatus 4 (A4), from left to right: mouthpiece, in-line filter, elbow connector, pneumotachograph, coupling device. The same lid is used for apparatus 1 and 2 , and the same lid for 3 and 4 . Despite different connector diameters for the two lids, the inside diameter of the four connections to the coupling device is nearly the same, being represented by the inside diameter $(26 \mathrm{~mm})$ of the lid connector in apparatus 1 and 2 and by the inside diameter of the pneumotachograph in apparatus 3 and $4(25 \mathrm{~mm})$.

the screen pneumotachograph. The difference between A2 and A3 represents the screen pneumotachograph's efficacy as a respiratory filter as compared with the Pall PF30SJE. A p-value $<0.05$ was considered significant.

\section{RESULTS}

Visual inspection of the blood agar plates in situation A1 showed that vapour condensation occurred on the entire surface of the plate. A summary of the results is given in figure 4 .

Of the 40 plates obtained with each apparatus, 13 were sterile with A1 (range 0-679 CFUs), 25 with A2 (range 0-49 CFUs), 30 with A3 (range 0-35 CFUs) and 39 with A4 ( $\mathrm{p}<0.001$; Cochran $\mathrm{Q}$ test). A1 produced significantly fewer sterile plates than A2 $(\mathrm{p}<0.01 ;$ McNemar's test) as did A3 versus A4 $(\mathrm{p}<0.01)$, A2 versus A4 $(p<0.001)$ and A1 versus $\mathrm{A} 3(\mathrm{p}<0.001)$ but there was no significant difference between $A 2$ and $A 3(p=0.07)$.

The five plates with $>150$ CFUs $(679,342,279,277$ and 240) were obtained with A1. The number of CFUs was affected by the apparatus $(\mathrm{p}<0.001$; Friedman test). A1 had significantly more CFUs than A2 ( $<<0.001$; Wilcoxon paired-sample test), as did A3 versus A4 $(\mathrm{p}<0.01)$, A1 versus $\mathrm{A} 3(\mathrm{p}<0.001)$ and $\mathrm{A} 2$ versus $\mathrm{A} 4(\mathrm{p}<0.001)$ but the contamination in $\mathrm{A} 2$ and $\mathrm{A} 3$ was not significantly different $(p=0.97)$. There was only one $C F U$ in the one positive plate of A4. For this subject, there were 679 CFUs in A1, 21 CFUs in A2 and 31 CFUs in A3. 


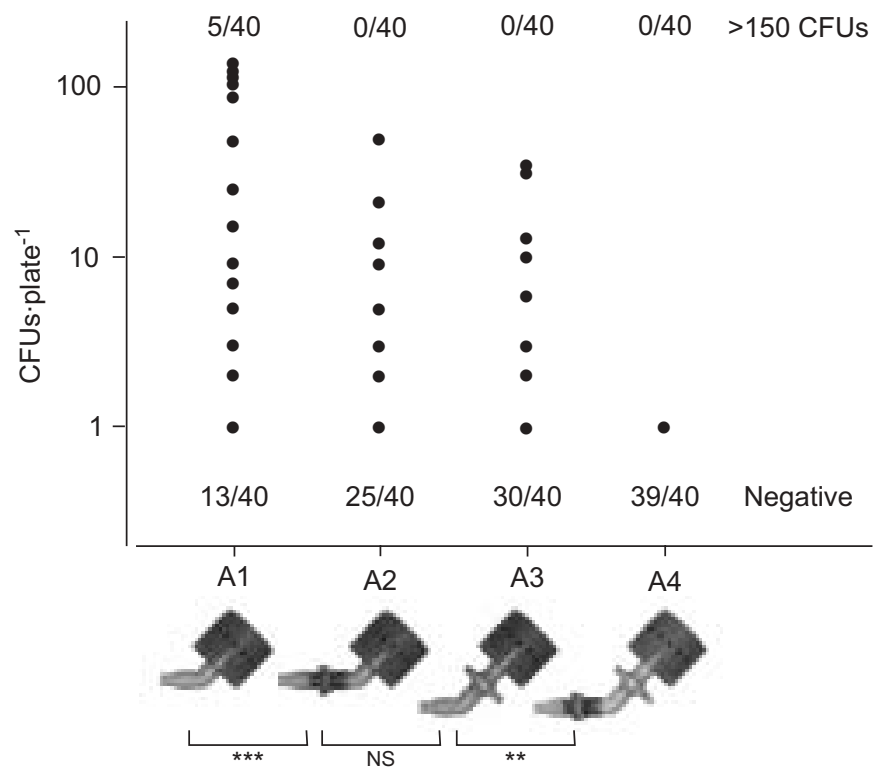

FIGURE 4. Number of colony forming units (CFUs) in positive plates. The number of negative plates is indicated at the bottom of the figure and the number of plates with $>150$ CFUs is indicated at the top of the figure. $* *$ : $p<0.01$; $* * *$ : $p<0.001$; Ns: Nonsignificant.

\section{DISCUSSION}

The present results indicate that with healthy subjects performing maximal expirations according to the ATS/ERS standards for studying the expiratory flow-volume curve, contamination occurs downstream from an in-line filter supposed to prevent cross-contamination during pulmonary function testing. These results are in accordance with the study of LEEMING et al. [19] who showed that the same filter allowed the transmittance of approximately one-third of expired CFUs in 37 patients with respiratory diseases, the highest CFU count being obtained in cystic fibrosis patients. Accordingly, as pointed out by the ATS/ERS Task Force: "The use of in-line filters does not eliminate the need for regular cleaning and decontamination of lung function equipment" [7].

Despite the fact that all subjects were free from current or past respiratory disease, the current authors observed a large intersubject variability in the number of CFUs, with five subjects producing $>150$ CFUs (one producing $>500$ ) in the control condition while 13 subjects produced no contamination of the agar plate. Such variability has already been mentioned [11, 23] and may be linked to high inter-subject variability in the number of small-size particles produced during mouth breathing [24], possibly relating to the physical properties of the lung lining fluid [25]. Several mechanisms have been described to explain the retention properties of filters in terms of particle size. Direct interception filters large particles $(>1 \mu \mathrm{m})$, inertial impaction removes smaller particles $(0.5-1.0 \mu \mathrm{m}$ in diameter) by collision within the filter material and very small particles, because of their Brownian motion, can be trapped by diffusional interception. Other properties, hydrophobic or electrostatic, are held to improve a filter's efficiency.

Contamination after the filter is similar to that observed downstream from a screen pneumotachograph whose resistance to flow is in the same order of magnitude. Therefore, the Pall filter is no better than a screen pneumotachograph. The resistance of the Pall PF30S was measured as $0.56 \mathrm{~cm} \cdot \mathrm{H}_{2} \mathrm{O} \cdot \mathrm{s} \cdot \mathrm{L}^{-1}$ at $12 \mathrm{~L} \cdot \mathrm{s}^{-1}$ [12], close to the value given by the manufacturer $\left(0.6 \mathrm{~cm} \cdot \mathrm{H}_{2} \mathrm{O} \cdot \mathrm{s} \cdot \mathrm{L}^{-1}\right)$. The resistance of the same screen pneumotachograph used in the same setting (non-heated) can be estimated from the graphical representation at $\sim 0.7$ to $0.8 \mathrm{~cm} \cdot \mathrm{H}_{2} \mathrm{O} \cdot \mathrm{s} \cdot \mathrm{L}^{-1}$ at $12 \mathrm{~L} \cdot \mathrm{s}^{-1}$ [22]. The pneumotachograph used was nonheated in order to keep a similar manipulation time for the four apparatuses. However, a high temperature around the proximal tubing could inhibit bacterial growth [6]; therefore, heating the pneumotachograph, while not changing its efficiency as a bacterial filter, could reduce the bacterial growth and the risk of inter-subject contamination.

In one experimental design, the SensorMedics MicroGard $₫$ Filter (SensorMedics Corp., Yorba Linda, CA, USA) and the KOKO MOE $®$ filter (KK; Pulmonary Data Service Instrumentation, Inc., Louisville, CO, USA) have demonstrated greater bacterial removal efficiency than the Pall PF30S [20]. However, the measured resistance of such filters is found to be slightly higher [26] and the experimental design is far from the conditions of natural contamination occurring in pulmonary function testing. In particular, the droplets produced by this experimental condition $(3-5 \mu \mathrm{m})$ are much larger than the droplets $(<1 \mu \mathrm{m})$ produced by normal breathing [25] and even coughing [24]. During standard spirometry, the SensorMedics filter was considered no different from the Pall filter [27].

The controversy over using in-line filters for lung function testing resembles the one over the use of filters in mechanical ventilation [28]: it is a balance between the advantages and disadvantages. Several issues must be considered; some, but not all of which are related to hygiene. 1) As there is substantial inter-individual variability in the number of CFUs expired during forced expiration, the potential for any one person to contaminate the equipment is unpredictable. 2) In-line filters reduce but do not eliminate contamination of the downstream equipment. 3) The ability of in-line filters to prevent crosscontamination has not been demonstrated; however, neither has it been demonstrated that cross-infection has occurred due to the use of pulmonary function testing equipment. 4) In-line filters introduce an undesirable resistance to flow. 5) Use of an in-line filter has a statistical effect on the results of certain lung function parameters, at least in those nonheated pneumotachographs. 6) The widely used pneumotachograph (screen or Fleisch type) may be subject to mucus deposition or vapour condensation that could considerably alter the flow and volume measurements, giving rise to a gross overestimation of respiratory parameters [29]. 7) In-line filters can behave as heat and moisture exchange filters [30]. Thus, the body, temperature, ambient pressure, saturated with water vapour (BTPS) corrections factor for the expiratory gas should be adapted to the actual gas condition in the pneumotachograph resulting from the use of the filter and its heat and moisture exchange characteristics. The error might be as large as $10 \%$ [21] and will not be taken in account when calibrating with the filter in place. At the present time, this aspect would seem not to have been taken into account by the lung equipment manufacturers, as for most equipment the presence of a filter is at best mentioned to correct the resistance measurements or 
the dead space of the equipment. Different heat and moisture exchange characteristics and resulting BTPS correction or different methods of BTPS correction may explain why, independently of the resistance, spirometry results are [12, 13], or are not [3], affected by the presence of a filter. One advantage of this potential characteristic is that the need to heat the pneumotachograph to prevent vapour condensation can be reconsidered [31].

In conclusion, the present study shows that, combined with the screen pneumotachograph, a low resistance filter virtually protects complex downstream equipment from contamination. This leads us to believe that in clinical situations where such contamination of complex equipment could occur, the use of an in-line filter might be justified, provided that measurements remain accurate, that the question of body, temperature, ambient pressure, saturated with water vapour correction is solved, while keeping in mind that the pneumotachograph must be decontaminated. Conversely, such a filter seems to have limited efficiency in terms of bacterial retention, no better in fact than the pneumotachograph itself. In the absence of complex equipment, the current authors therefore believe that, where necessary, decontamination of the pneumotachograph is a preferable solution, if possible, unless other advantages of the filter in terms of mucus retention or heat exchange are demonstrated.

\section{ACKNOWLEDGEMENTS}

The authors would like to thank A. Lemarié and Y. Liégard (University Hospital of Caen, Caen, France) for technical assistance throughout this project and to P. Thibon (University Hospital of Caen, Caen, France) for helpful advice in the statistical analysis.

\section{REFERENCES}

1 Govan JR, Brown PH, Maddison J, et al. Evidence for transmission of Pseudomonas cepacia by social contact in cystic fibrosis. Lancet 1993; 342: 15-19.

2 LiPuma JJ, Dasen SE, Nielson DW, Stern RC, Stull TL. Person-to-person transmission of Pseudomonas cepacia between patients with cystic fibrosis. Lancet 1990; 336: 1094-1096.

3 Rogers D, Doull IJ. Effect of a microaerosol filter on spirometry in children with cystic fibrosis. Acta Paediatr 2002; 91: 1257-1259.

4 Isles A, Maclusky I, Corey M, et al. Pseudomonas cepacia infection in cystic fibrosis: an emerging problem. J Pediatr 1984; 104: 206-210.

5 Hazaleus RE, Cole J, Berdischewsky M. Tuberculin skin test conversion from exposure to contaminated pulmonary function testing apparatus. Respir Care 1981; 26: 53-55.

6 Burgos F, Torres A, Gonzalez J, Puig de la Bellacasa J, Rodriguez-Roisin R, Roca J. Bacterial colonization as a potential source of nosocomial respiratory infections in two types of spirometer. Eur Respir J 1996; 9: 2612-2617.

7 Miller MR, Crapo R, Hankinson J, et al. General considerations for lung function testing. Eur Respir J 2005; 26: 153-161.

8 Rutala DR, Rutala WA, Weber DJ, Thomann CA. Infection risks associated with spirometry. Infect Control Hosp Epidemiol 1991; 12: 89-92.
9 Depledge $\mathrm{MH}$, Barrett A. Aseptic techniques for lung function testing. J Hosp Infect 1981; 2: 369-372.

10 Clausen JL. Lung volume equipment and infection control. ERS/ATS Workshop Report Series. European Respiratory Society/American Thoracic Society. Eur Respir J 1997; 10: 1928-1932.

11 Kirk YL, Kendall K, Ashworth HA, Hunter PR. Laboratory evaluation of a filter for the control of cross-infection during pulmonary function testing. J Hosp Infect 1992; 20: 193-198.

12 Johns DP, Ingram C, Booth $\mathrm{H}$, Williams TJ, Walters EH. Effect of a microaerosol barrier filter on the measurement of lung function. Chest 1995; 107: 1045-1048.

13 Fuso L, Accardo D, Bevignani G, Ferrante E, Dellacorte A, Pistelli R. Effects of a filter at the mouth on pulmonary function tests. Eur Respir J 1995; 8: 314-317.

14 Kamps AWA, Vermeer K, Roorda RJ, Brand PLP. Effect of bacterial filters on spirometry measurements. Arch Dis Child 2001; 85: 346-347.

15 Becquemin $\mathrm{MH}$, Camus F, Lucet JC, et al. Recommendations of the usefulness and efficacy of filters for respiratory function testing. Integral recommendations solicited from experts and validated by the CLIN-central of 28 April 1997. Rev Mal Respir 1999; 16: 585-588.

16 Kendrick AH, Johns DP, Leeming JP. Infection control of lung function equipment: a practical approach. Respir Med 2003; 97: 1163-1179.

17 Zhang Y. High justification for universal stringent precautions in lung function testing. Respir Med 2005; 99: 1064-1066.

18 Kendrick AH, Johns DP, Leeming JP. Author's reply. Respir Med 2005; 99: 1067.

19 Leeming JP, Pryce-Roberts DM, Kendrick AH, Smith EC. The efficacy of filters used in respiratory function apparatus. J Hosp Infect 1995; 31: 205-210.

20 Canakis AM, Ho B, Ho S, Kovach D, Matlow A, Coates AL. Do in-line respiratory filters protect patients? Comparing bacterial removal efficiency of six filters. Pediatr Pulmonol 2002; 34: 336-341.

21 Miller MR, Hankinson J, Brusasco V, et al. Standardisation of spirometry. Eur Respir J 2005; 26: 319-338.

22 Johns DP, Ingram CM, Khov S, Rochford PD, Walters EH. Effect of breathing circuit resistance on the measurement of ventilatory function. Thorax 1998; 53: 944-948.

23 Leeming JP, Kendrick AH, Pryce-Roberts D, Smith D, Smith EC. Use of filters for the control of cross-infection during pulmonary function testing. J Hosp Infect 1993; 23: 245-246.

24 Papineni RS, Rosenthal FS. The size distribution of droplets in the exhaled breath of healthy human subjects. J Aerosol Med 1997; 10: 105-116.

25 Edwards DA, Man JC, Brand P, et al. Inhaling to mitigate exhaled bioaerosols. Proc Natl Acad Sci USA 2004; 101: 17383-17388.

26 Squires P, Norgard E. A flow resistance comparison of pulmonary function testing filters. Pulmonary Data Services Inc., Louisville USA, 1995; company communication.

27 Clayton N, Collyns T, Egan J, Isalska B, Standbridge T, Woolcock A. Evaluation of effectiveness of four bacterial filters during pulmonary function testing. Thorax 1995; 50: A65. 
28 Lawes EG. Hidden hazards and dangers associated with the use of HME/filters in breathing circuits. Their effect on toxic metabolite production, pulse oximetry and airway resistance. Br J Anaesth 2003; 91: 249-264.

29 Townsend MC, Hankinson JL, Lindesmith LA, Slivka WA, Stiver G, Ayres GT. Is my lung function really that good? Flow-type spirometer problems that elevate test results. Chest 2004; 125: 1902-1909.
30 Turnbull D, Fisher PC, Mills GH, Morgan-Hughes NJ. Performance of breathing filters under wet conditions: a laboratory evaluation. Br J Anaesth 2005; 94: 675682.

31 Miller MR, Pedersen OF, Sigsgaard T. Spirometry with a Fleisch pneumotachograph: upstream heat exchanger replaces heating requirement. J Appl Physiol 1997; 82: 1053-1057. 